CARNETS DE Carnets de géographes

GÉOGRAPHES.

$3 \mid 2011$

Les géographies des enfants et des jeunes

\title{
Géographies des enfants et des jeunes
}

Sonia Lehman-Frisch et Jeanne Vivet

\section{OpenEdition}

Journals

Édition électronique

URL : http://journals.openedition.org/cdg/2074

DOI : $10.4000 /$ cdg. 2074

ISSN : 2107-7266

Éditeur

UMR 245 - CESSMA

Référence électronique

Sonia Lehman-Frisch et Jeanne Vivet, " Géographies des enfants et des jeunes », Carnets de

géographes [En ligne], 3 | 2011, mis en ligne le 01 décembre 2011, consulté le 24 septembre 2020.

URL : http://journals.openedition.org/cdg/2074 ; DOI : https://doi.org/10.4000/cdg.2074

\section{(c) (1) ()}

La revue Carnets de géographes est mise à disposition selon les termes de la Licence Creative Commons Attribution - Pas d'Utilisation Commerciale - Pas de Modification 4.0 International. 


\title{
GEOGRAPHIES DES ENFANTS ET DES JEUNES
}

\author{
SONIA LEHMAN-FRISCH \\ MRTE \\ Université de Cergy-Pontoise \\ sonia.frisch@ens.fr \\ JEANNE VIVET \\ Institut des Sciences sociales \\ Université de Lisbonne \\ jeanne.vivet@gmail.com
}

L'introduction des actes d'un récent colloque sur " les enfants et les jeunes dans les espaces du quotidien" (Danic et al., 2010) affirmait que "la question du rapport des enfants aux espaces (...) a été peu investiguée " (David, 2010 : 9). Partant de ce constat, ce numéro spécial des Carnets de Géographes s'est donné pour objectif de contribuer à valoriser les enfants comme sujets de recherche pour les sciences sociales, et de montrer la nécessité de développer les travaux sur les rapports des enfants au territoire en France.

L'appel à contributions, lancé en janvier 2011, proposait ainsi d'interroger les " géographies des enfants et des jeunes" dans une double perspective. D'une part, il s'agissait de questionner la place des enfants et des jeunes dans l'espace (urbain ou rural), en analysant leur répartition ainsi que la géographie des lieux qui leur sont dédiés, son évolution dans le temps et ses inégalités dans l'espace. II invitait à interroger les impacts des différentes politiques publiques (dans le domaine scolaire, du logement, de l'aménagement, etc.) menées en milieu urbain ou rural sur les territoires des enfants. D'autre part, l'appel à contributions soulignait la nécessité de mettre en avant la perspective des enfants euxmêmes ou, autrement dit, les géographies qu'ils produisent. Comment, en particulier, construisent-ils leurs territoires quotidiens, leurs ancrages, leurs sociabilités et leurs mobilités, par leurs pratiques et leurs représentations ? Comment les enfants habitent-ils et cohabitent-ils dans leur quartier, et dans leur ville ? Comment caractériser les mobilités des enfants et des jeunes dans la ville et leur autonomie spatiale ? Comment leurs territoires et leurs mobilités sont-ils structurés par des différences en termes de milieu social, de genre, d'origine ethnique, d'âge, et également en fonction des environnements dans lesquels ils vivent (espaces urbains, espaces ruraux) ? Comment imaginent-ils d'autres espaces, plus 
vastes ou plus lointains ? Comment les enfants de migrants vivent-ils la migration (qu'ils l'aient expérimentée directement, ou indirectement à travers leurs parents) et quels impacts l'expérience de la migration a-t-elle sur leurs rapports au(x) territoire(s)?

Le succès de cet appel à contributions, auquel de nombreux jeunes chercheurs (à côté de quelques chercheurs confirmés) ont répondu, signale clairement l'émergence de ces questionnements dans les sciences sociales françaises contemporaines. De plus, il a montré que cet intérêt nouveau pour les rapports des enfants et des jeunes à l'espace s'étend bien au-delà de la géographie: de nombreuses propositions relevaient en effet de disciplines aussi variées que l'urbanisme, la sociologie, les sciences de l'éducation, les sciences de l'information ou encore l'acoustique, ce qui traduit le caractère fondamentalement pluridisciplinaire des géographies des enfants et des jeunes.

Avant de revenir sur les grandes lignes qui se dégagent de cet ensemble d'articles et de présenter succinctement les apports de chacun d'entre eux, cet éditorial commence par esquisser une revue des travaux francophones et anglophones portant sur la question des rapports des enfants et des jeunes au territoire afin d'offrir un cadre théorique aux contributions de ce numéro.

\section{Les géographies des enfants et des jeunes, un champ de recherche émergent}

Les rapports des enfants et des jeunes au territoire constituent un domaine de recherche encore sous-exploré dans les sciences sociales francophones aujourd'hui. Pourtant, un certain nombre de travaux avaient ouvert la voie dans les années 1970 et 1980, à la fois en France et aux Etats-Unis. Cette section retrace les développements de ces recherches depuis quatre décennies et montre leur relative marginalisation à partir des années 1990 en France, alors même que les Children's Studies émergeaient comme sous-discipline spécifique dans les pays anglophones. ${ }^{1}$

\section{L'éveil des sciences sociales francophones aux enfants}

C'est dans les années 1970 qu'apparaissent en France les premiers travaux étudiant les enfants et les jeunes dans leurs relations au territoire. En géographie notamment, un certain nombre de travaux s'intéressent ainsi aux représentations de l'espace des enfants, dans le sillage de la "géographie de la perception ". Ce courant de la géographie, qui s'est développé autour de chercheurs comme Antoine Bailly, Jean-Paul Guérin, Hervé Gumuchian ou Bertrand Debarbieux pour n'en citer que quelques uns, s'est inspiré de la psychologie cognitive (Piaget et Inhelder, 1972) et de la notion d'espace vécu (Frémont, 1976) : pour eux, l'espace, loin d'être objectif et rationnel, est le produit d'une construction mentale ; ils se donnent donc pour objectif de saisir les représentations de l'espace (individuelles et collectives), en s'appuyant notamment sur les techniques des cartes mentales développées aux Etats-Unis par Kevin Lynch (1960), Peter Gould et Rodney White (1974). Dans cette

\footnotetext{
${ }^{1}$ Ce bref état de la recherche sur les enfants et l'espace dans la littérature francophone et anglophone doit beaucoup aux réflexions menées par S. Lehman-Frisch, J.-Y. Authier et F. Dufaux dans le cadre d'une enquête en cours portant sur les manières d'habiter et de cohabiter des enfants et de leurs parents dans différents types de quartiers de Paris, Londres et San Francisco.
} 
perspective, reconnaissant que " chaque individu est unique dans sa relation au monde mais appartient aussi à un groupe socio-culturel, à un groupe qui nourrit sa vision " (André et al. 1989 : 162, cité dans Breux et Reuchamps, 2010 : 9), et que les cartes mentales (et les représentations qu'elles expriment) évoluent sensiblement avec l'âge, des chercheurs se sont plus particulièrement penchés sur les représentations des enfants. Ils ont ainsi étudié la façon dont ils conçoivent différents objets géographiques (Sgard et Hoyaux, 2006) : bien que la plupart de ces recherches portent sur les représentations de la ville (Bertrand et Metton, 1972 ; Metton, 1976 ; Ferras, 1978 ; Lugadet-Agraz, 1989 ; plusieurs articles dans Callenge et al., 1997), d'autres travaux ont aussi été réalisés sur la montagne (Masson-Vincent, 1990), la région ou le monde (André et al., 1989). Dans l'ensemble, ces recherches ont cependant pour point commun de s'intéresser aux mécanismes de construction des représentations dans une perspective cognitive davantage qu'à leurs dimensions symboliques (Bonin, 2004).

À côté de ces travaux, on recense également quelques travaux pionniers de sociologues s'intéressant aux enfants dans leurs relations à l'espace, principalement pour dénoncer l'inadaptation de l'environnement urbain à la condition enfantine. C'est le cas de Rose-Marie de Casabianca, qui, dès 1959, étudie "la vie extrascolaire des enfants " dans un quartier parisien populaire du $9^{\text {ème }}$ arrondissement, la paroisse de Notre-Dame de Lorette, préconisant d'améliorer la ville pour réformer les "pathologies sociales". À la fin des années 1970, Marie-José Chombart de Lauwe réalise avec son équipe une enquête pour le Ministère de la Qualité de la vie (1987) : comparant les pratiques d'enfants de 6 à 14 ans dans différents environnements (un quartier ancien de Paris, des quartiers de villes nouvelles et un village de Lozère), elle souligne les "malajustements entre les enfants et l'espace construit " (Chombart de Lauwe, $1977: 1$ ) et tout particulièrement ceux des "vieux quartiers " qui n'offrent rien pour satisfaire les besoins en loisir des enfants. Mais dans l'ensemble, ces travaux sont restés relativement marginaux dans les sciences sociales.

Aujourd'hui, un certain nombre de chercheurs, en France, s'intéressent aux rapports des enfants et des jeunes à leur territoire. C'est à des sociologues que I'on doit en particulier tout un ensemble de travaux récents portant en particulier sur les adolescents (Galland, 2006), et plus spécifiquement sur les jeunes de milieux populaires, résidant dans des quartiers d'habitat social localisés en banlieue (Lepoutre, 2001 ; Beaud, 2002 ; Kokoreff, 2003) ou, de façon moins visible, dans des bourgs ruraux industriels (Rénahy, 2005). Ces productions, montrent que le quartier ou le village, loin d'être un simple décor ou un handicap, constitue souvent pour ces adolescents ou ces jeunes, un " espace ressource » et un lieu important de socialisation.

Les recherches portant sur des enfants plus jeunes sont plus rares. Dans le contexte de l'émergence d'une " sociologie de l'enfance » (Sirota, 2006) à la marge de la sociologie de la famille et de la sociologie de l'éducation, certains travaux se sont cependant penchés sur la relation des enfants à différents types d'espaces : la salle de classe, la cour de recréation, l'établissement (Delalande, 2001, 2003, 2010 ; Rayou, 2006), ou, dans une moindre mesure, le logement (Sirota, 1998 ; Lignier, 2010), dans le prolongement ici d'autres travaux plus anciens sur la chambre d'enfant (Neitzert, 1990) et sur les espaces des relations entre parents et enfants dans le logement (De Singly, 1998). Quelques productions de sociologues ou de géographes sont plus directement centrées sur le rapport à la ville des enfants et des jeunes. Un certain nombre d'entre elles, qui portent surtout sur des villes de pays en 
développement, s'intéressent ainsi aux « enfants des rues » (Tessier, 2005 ; Morelle, 2007). Ce sont finalement les psychologues, parfois associés à des géographes, qui, dans la lignée des travaux de Jean Piaget, sont les plus nombreux à s'intéresser aux enfants : certains d'entre eux analysent les modalités de développement des compétences spatiales des enfants dans une perspective cognitive (Ramadier et Depeau, 2010 ; Enaux et Legendre, 2010); d'autres se penchent sur la manière dont les connaissances de l'espace (urbain) des enfants s'articulent avec leurs pratiques, leurs mobilités et leur autonomie de déplacement (Depeau, 2003, 2008 ; Tsoukala, 2007 ; Legendre 2010). Les travaux sur les manières d'habiter et de cohabiter des enfants dans la ville sont encore rares (Authier et LehmanFrisch, à paraître).

Mais au total, les travaux sur les rapports à l'espace des enfants et des jeunes sont encore relativement peu développés dans les sciences sociales francophones ${ }^{2}$, et ce déficit offre un contraste saisissant avec la vitalité de ce champ dans la littérature scientifique anglophone, comme l'avaient déjà constaté Rachel Gurevitz et André-Louis Sanguin (2001).

\section{Le « baby boom » des Children's Geographies}

Les racines des Children's Geographies anglophones remontent elles aussi aux années 1970. C'est à cette époque que se sont développés deux ensembles de travaux distincts, préfigurant deux grands types d'approches qui persistent encore aujourd'hui dans les recherches sur les enfants et l'espace. D'un côté, James Blaut et David Stea, chercheurs en psychologie environnementale à l'Université de Clark, mettent en place le " Place Perception Project " visant à développer la compréhension des processus cognitifs d'appréhension de l'espace par les enfants et de développement de leurs compétences cartographiques dans une perspective éducative. Ainsi, en utilisant différentes méthodes visuelles, au premier rang desquelles, bien sûr, les cartes mentales, plusieurs chercheurs ont montré les variations des modalités de la connaissance de l'espace en fonction de l'âge et du sexe des enfants tout en remettant en cause l'hypothèse piagétienne des étapes du développement de l'enfant (Blaut et Stea, 1971; Matthews, 1984, 1985, 1992). D'un autre côté, William Bunge s'intéresse à la question des pratiques spatiales et de l'attachement des enfants à l'espace urbain à Détroit : ses " expéditions géographiques " visent à montrer l'oppression spatiale subie par les enfants dans leur environnement quotidien, et à mettre en évidence que les enfants sont finalement les principales victimes des forces politiques, économiques et sociales qui façonnent l'espace urbain (Bunge, 1973). Moins ouvertement polémiques, quelques autres chercheurs s'engagent sur la voie d'explorer les pratiques urbaines des enfants aux Etats-Unis (Ward, 1978 ; Hart, 1979) ou ailleurs (Lynch, 1977).

C'est à partir des années 1990 seulement que les recherches sur le rapport des enfants au territoire (et à la ville en particulier) connaissent un développement véritablement spectaculaire. Deux facteurs sociétaux et intellectuels contribuent à l'essor de ces travaux. D'abord, la Convention Internationale des Droits de l'Enfant est adoptée en 1989, et, dans

\footnotetext{
2 II faut ici cependant mentionner les recherches québécoises qui occupent une place particulière, au carrefour des mondes universitaires francophone et anglophone, et à qui l'on doit un certain nombre de publications en français mais s'inscrivant clairement dans le champ des recherches anglophones (voir par exemple Cloutier et Torres, 2010).
} 
son sillage, les instances internationales insistent de manière récurrente sur la nécessaire prise en compte des enfants dans la mesure du bien-être des sociétés et sur leur place dans la ville notamment (alors que les enfants comptent aujourd'hui pour un peu moins du tiers de la population urbaine mondiale). En propulsant les enfants sur le devant de la scène internationale, ces événements contribuent à légitimer l'intérêt scientifique pour une population jusque-là négligée par la recherche (McKendrick, 2000).

Parallèlement, les années 1990 sont le temps d'une vaste réflexion scientifique sur le concept d'enfant, qui a nourri le renouvellement des travaux sur la question des enfants et des jeunes. Ainsi, s'appuyant sur les travaux de Philippe Ariès, qui ont montré la façon dont la conception de l'enfance comme un âge spécifique de la vie, distinct de l'âge adulte, a historiquement émergé au $16^{\text {ème }}$ siècle et s'est décisivement consolidée à l'époque des Lumières (Ariès, 1962), des chercheurs ont contesté une conception naturaliste de l'enfant qui imprègne encore largement les discours contemporains. Ils ont montré que loin d'être un donné biologique, l'enfance est en réalité une construction sociale, qui varie sensiblement selon les milieux sociaux, les sociétés, et les périodes de l'histoire (Prout et James, 1990 ; Jenks, 1996). Ainsi, la question même des limites de l'enfance demeure problématique puisque soumise à ces variations spatiales et temporelles. Ces analyses épistémologiques, en posant les enfants comme êtres à part entière (et non comme êtres en devenir, devant être soumis aux forces de socialisation pour pouvoir atteindre la complétude de l'âge adulte), les placent au rang de véritables acteurs sociaux, et soulignent la nécessité de les mettre au centre des recherches en tant que tels. De plus, ces réflexions ont conduit à contester la notion d'enfant comme étant universelle et de nombreux chercheurs ont insisté sur l'importance de prendre en compte les différenciations sociales et spatiales des enfants pour saisir la complexité et la multiplicité de leurs vies quotidiennes et de leurs rapports à l'espace (Aitken 1994 ; Matthews et Limb, 1999 ; Holloway et Valentine, 2000) :

Children come in all shapes and sizes and may be distinguished along various axes of gender, race, ethnicity, ability, health and age. (...) We emphasize the need to recognize the importance of 'multiple childhoods' and the sterility of the concept of the 'universal child'3 (Matthews et Limb, $1999: 65$ ).

Dans ce contexte épistémologique renouvelé, et encouragées de surcroît par le lancement de vastes programmes de recherches nationaux (I'ESRC au Royaume-Uni, et la NSF aux EtatsUnis) et internationaux (UNESCO), les recherches se sont considérablement développées depuis la fin des années 1990, au point de se constituer en un champ spécifique, les Children's Geographies, qui possède ses propres colloques, ses ouvrages de référence (par exemple Holloway et Valentine, 2000; Chawla, 2002; O'Brien et Christensen, 2003 ; Valentine, 2004 ; Holt, 2011), et ses revues spécialisées (voir par exemple le renouveau de la revue Children, Youth and Environment en 1992 et la création d'une revue intitulée Children's Geographies en 2003). Ces travaux sur les enfants accordent une attention toute particulière à la dimension spatiale (Holloway et Valentine, 2000), ou, pour le dire autrement, les Children's Geographies visent à mettre en lumière les multiples façons dont

\footnotetext{
${ }^{3}$ " Les enfants ont tous des silhouettes et des tailles différentes et ils se distinguent les uns des autres en fonction de leur genre, de leur race, de leur ethnicité, de leurs capacités, de leur santé et de leur âge. (...) Nous insistons sur la nécessité de reconnaître l'importance de 'la multiplicité des enfances' et la stérilité du concept d' 'enfant universel' ».
} 
l'espace structure les vies quotidiennes des enfants et des jeunes, et dont, inversement, les enfants réinterprètent et construisent leur propre territoire.

Les divers états de la recherche produits ces dernières années distinguent plusieurs axes structurant la sous-discipline aujourd'hui (Holloway et Valentine, 2000; Christensen et O'Brien, 2003; Horton et al., 2008; Holloway et Pimlott-Wilson, 2010). Un premier ensemble de travaux, dans la lignée du "Place Perception Project " des années 1970, continue de s'intéresser à la connaissance de l'espace et aux compétences cartographiques des enfants. James Blaut, David Stea et leurs collègues, par exemple ont conduit une recherche auprès d'enfants de 3 à 5 ans dans différents contextes urbains et ruraux de plusieurs pays du Nord et du Sud, dont les résultats ont établi que les jeunes enfants étaient capables d'interpréter des photographies aériennes et des cartes simples pour s'orienter ; ils en concluent que les ambitions pédagogiques devraient être revues à la hausse concernant cette tranche d'âge, et les méthodes d'enseignement profondément repensées (Blaut et al.). Ces approches cognitives, pour minoritaires qu'elles soient devenues dans les Children's Studies, demeurent tout à fait pertinentes et des chercheurs mettent en garde contre leur éventuelle marginalisation au sein de la sous-discipline (Holloway et Pimlott-Wilson, 2010).

Avec l'émergence de l'approche sociologique de l'enfant et la reconnaissance que les enfants constituent des acteurs sociaux en tant que tels, les recherches ont davantage prêté attention aux perspectives des enfants eux-mêmes sur leurs territoires (à leurs pratiques et à leurs représentations spatiales), au sens et aux valeurs qu'ils y accordent et aux formes d'attachement qu'ils éprouvent. Ce deuxième ensemble de travaux, le plus fourni au sein des Children's Geographies aujourd'hui, s'est ainsi penché sur le rapport des enfants à différents types de territoires du quotidien. Le logement est mis en avant comme un lieu important de la définition des relations de pouvoir entre les adultes et les enfants et des négociations sur les limites de l'intérieur et de l'extérieur - autrement dit, les pratiques spatiales qui se définissent au sein du logement sont fortement liées aux pratiques spatiales en dehors du logement, dans le quartier ou dans la ville (Aitken, 1994 ; Sibley, 1995 ; Christensen et al., 2000; Halldén, 2003). Les institutions scolaires et périscolaires constituent un deuxième type de lieu à partir desquels les rapports des enfants à l'espace sont analysés: des recherches montrent l'importance de ces lieux pour l'apprentissage, analysent la façon dont ils spatialisent le contrôle des adultes sur les enfants et les manières dont ils contribuent à reproduire les différences (de genre, de milieu social, etc.) entre les enfants (Aitken, 1994 ; Smith et Barker, 2000 ; Kraflt, 2006 ; Hollingworth, et al., 2011). Audelà du logement et de l'école, de nombreux travaux s'intéressent aux pratiques urbaines des enfants et des jeunes. Ils tendent à montrer le déclin de la présence des enfants dans les espaces publics, la préoccupation croissante de la société pour la sécurité des enfants ayant entraîné l'enfermement des enfants et la privatisation, l'institutionnalisation et I'archipélisation de leurs territoires dans les villes des pays développés (Holloway, Valentine, 2000 ; Christensen, O'Brien, 2003 ; Valentine, 2004 ; Karsten et Van Vilet, 2006 ; Holt, 2011 ${ }^{4}$ ). Dans le prolongement de ces travaux sur les enfants et la ville, des chercheurs se sont

\footnotetext{
${ }^{4}$ On peut aussi mentionner ici l'ouvrage d'Annette Lareau (2003), qui donne de nombreux éléments sur les pratiques urbaines des enfants et leurs différenciations en fonction de leur milieu social, bien que cette question ne constitue pas la focale principale de la recherche.
} 
penchés sur la question de l'adaptation ou de l'inadaptation de l'environnement urbain au bien-être des enfants et prônent leur participation aux processus d'aménagement, dans une forte perspective appliquée (Chawla, 2002). Globalement, ces travaux se sont intéressés de façon privilégiée au Nord, et plus particulièrement aux pays du monde anglophone, bien que les recherches portant sur le Sud se soient récemment développées (Chawla, 2002 ; Katz, 2004).

Un troisième groupe de travaux, plus restreint, s'intéresse aux discours portant sur les rapports des enfants et des jeunes à l'espace (celui de la ville, de la campagne, de la nation...) : ils ont montré l'importance de mettre en lumière les idéologies sous-jacentes à ces discours, dans la mesure où ceux-ci influencent profondément les pratiques sociospatiales des enfants (Holloway et Valentine, 2000 ; Holloway et Pimlott-Wilson; 2011). Par exemple, Gill Valentine a montré la façon dont la conception des enfants est fondamentalement territorialisée en Grande-Bretagne: les enfants sont alternativement pensés comme des "petits anges " (des êtres vulnérables et qu'il faut protéger des dangers inhérents aux espaces publics) ou comme des "petits démons" (des êtres aux comportements déviants dans les espaces publics). Cette conception duale et contradictoire des enfants repose ainsi sur l'idée que la place des enfants est à la maison et non dans les espaces publics (Valentine, 2004).

Un quatrième ensemble de recherches met l'accent sur d'autres dimensions et d'autres échelles de la question du rapport des enfants à l'espace (Holloway et Pimlott-Wilson, 2011). Ainsi, certaines se sont penchées sur le corps en montrant que l'expérience spatiale des enfants est profondément sensible, et que leurs représentations de l'espace sont informées par leurs perceptions, leurs émotions, leurs performances physiques et leurs pratiques (O’Brien, 2000 ; Woodyer ; 2008, Colls et Hörschelmann, 2009). D'autres ont porté une attention nouvelle à la question des mobilités et des migrations des enfants et analysent la façon dont elles marquent la vie quotidienne des enfants, et dont elles participent à la construction de leur identité (Van Blerk et Ansell, 2006 ; Wells et al., 2011 ; Hopkins et Hill, 2008; Hatfield, 2010; Holt et Costello, 2011). Enfin, tout en continuant à penser la dimension spatiale comme primordiale, des chercheurs ont commencé à réfléchir à l'impact des temporalités sur les vies quotidiennes des enfants, aux moments de transition, à l'intérieur et hors de l'enfance, et à la façon dont l'âge informe les expériences spatiales (Hopkins et Pain, 2007).

On peut enfin distinguer un dernier (vaste) ensemble de publications, qui s'appuie sur les réflexions sur le statut des enfants et des jeunes pour questionner les fondements éthiques et méthodologiques de leurs recherches. Les chercheurs des Children's Geographies manifestent en effet une forte propension à réfléchir, de façon parfois très critique, à leurs propres pratiques de recherche, au point que cette position réflexive est presque devenue un lieu commun de la sous-discipline aujourd'hui, contrairement à que l'on observe parfois dans les sciences sociales en général (Horton et al., 2008). Conscients que leurs enquêtés constituent un groupe particulièrement vulnérable, ils se sont largement interrogés sur les complexités éthiques inhérentes aux recherches menées auprès d'enfants et de jeunes, mettant en avant l'importance de clarifier un certain nombre d'enjeux : quel est le but de la recherche et quels intérêts sert-elle ? Quels sont les coûts et les bénéfices de la recherche pour les enquêtés? Comment l'anonymat et la confidentialité peuvent-ils être respectés? 
Les enquêtés ont-il la possibilité d'accepter ou de refuser de participer à la recherche? Comment éviter que les relations de pouvoir qui existent entre enfants et adultes n'influencent les résultats de la recherche? Quel impact la recherche a-t-elle sur les enquêtés a posteriori ? etc. (Aitken, 2001 ; Alderson et Morrows, 2004 ; Hopkins, 2010). Pour questionner les rapports des enfants à l'espace, les chercheurs ont développé des méthodes de recherches innovantes (quantitatives ou qualitatives), visant non seulement à se conformer à ces exigences éthiques, mais aussi à mettre à la disposition des enfants et des jeunes une grande variété d'outils pour rendre compte eux-mêmes de leurs interprétations de l'espace (entretiens individuels ou collectifs, méthodes visuelles, etc.) (Cele, 2006 ; White et al., 2010). Ils ont aussi expérimenté, dans cette perspective, des dispositifs promouvant la participation active des enquêtés à la recherche (la "Participatory Action Reseach "), laquelle est plus ou moins importante selon les démarches adoptées (prise de photographies par les enquêtés, réalisation de cartes mentales, rédaction de textes jusqu'à l'implication des adolescents dans la construction des questions de recherche) (Hart, 1992 ; Fine et al, 2003 ; Pain, 2004).

Au total, ce bref état de la littérature a révélé la relative ancienneté de l'intérêt des chercheurs anglophones pour les rapports des enfants et des jeunes au territoire, et l'émergence incontestable de ce champ dans les sciences sociales en France. Surtout, il a montré les grandes orientations de ces recherches aujourd'hui. C'est dans ce contexte que se positionnent les contributions de ce numéro spécial des Carnets de géographes.

\section{Des géographies des enfants et des jeunes en plusieurs Carnets}

\section{Les adolescents, la ville et l'école}

Les articles de ce numéro reflètent un certain nombre de tendances caractérisant les recherches (francophones et anglophones) sur le rapport des enfants et des jeunes au territoire. Ainsi, ils portent davantage sur les adolescents, collégiens ou lycéens ( 6 articles), que sur les enfants de moins de douze ans ( 3 articles). Les jeunes enfants sont encore une fois relativement marginalisés dans les géographies des enfants et des jeunes en général pour différentes raisons en partie évoquée précédemment (moindre visibilité des enfants par rapport aux jeunes dans les espaces publics; idée que plus les enfants sont jeunes, plus l'enquête est difficile, d'un point de vue tant méthodologique qu'éthique).

De plus, tout comme dans la majorité des recherches sur les géographies des enfants, c'est le rapport des enfants et des jeunes à l'espace urbain qui est au cœur de la majorité des contributions, qu'il s'agisse d'analyser leurs représentations urbaines et en particulier de l'espace public ou de mieux comprendre les mobilités d'adolescents de banlieue de métropoles. Cette prédominance des espaces urbains ne doit cependant pas occulter la grande diversité des terrains, à la fois parce que ce sont des villes de tailles très différentes (de la petite ville comme Lisieux aux banlieues de villes millionnaires que sont Paris ou Rio de Janeiro en passant par les espaces urbains et périurbains d'Ille-et-Vilaine) et aussi parce que deux articles portent sur des grandes villes du Sud, la ville nouvelle indienne de Chandigarh et Rio de Janeiro. En outre, si les articles privilégient les contextes urbains, ils les abordent en utilisant différentes échelles d'analyse : l'échelle micro (retenue par les auteurs 
s'intéressant aux espaces scolaires de l'école et du collège), l'échelle locale, en général confondue avec le périmètre de la ville ou de l'agglomération (utilisée par les auteurs cherchant à saisir les représentations spatiales des jeunes ou leurs mobilités quotidiennes), et enfin l'échelle départementale (utilisée par deux contributions portant sur le Rhône et l'Ille et Vilaine).

Enfin, et c'est là encore un point commun avec les recherches sur les rapports entre les enfants et les jeunes et l'espace, l'école constitue un lieu et un objet privilégiés de la recherche dans les différents articles. Plusieurs raisons expliquent cet intérêt des chercheurs pour l'école. D'une part, choisir l'école ou le lycée comme lieu de la recherche facilite le travail du chercheur, qui peut rencontrer ainsi facilement un grand nombre d'enfants. Les institutions scolaires constituent donc une voie d'entrée privilégiée pour le chercheur qui souhaite travailler auprès d'enfants ou de jeunes, un cadre commode permettant d'établir un contact avec des jeunes pour mener des entretiens (Hélène Pétry) ou leur faire réaliser des dessins durant le temps scolaire (Anna Dewaele). D'autre part, pour certains auteurs, l'école représente un cadre d'observation privilégié, comme c'est le cas d'Elisabeth Schneider pour qui l'observation en classe des pratiques scripturales des élèves constitue un élément essentiel de son dispositif d'enquête ethnographique. Nicolas Oppenchaim, quant à lui, a lancé un projet dans huit établissements scolaires qui comprenaient plusieurs dimensions : réalisation d'entretiens semi-directifs, de photographies et de textes sur les mobilités et initiation à la sociologie. Les élèves volontaires se sont faits apprentis chercheurs en réalisant des questionnaires sur la mobilité des jeunes auprès de leurs pairs et en exposant leurs résultats en classe. Le collège devient alors le lieu de fabrique de la recherche et les élèves ne sont plus seulement de simples objets, mais de véritables acteurs dans le processus de recherche. Enfin, d'autres auteurs s'intéressent à l'école en tant que territoire quotidien des enfants et des jeunes qui y passent une grande partie de leur temps, et pour qui c'est un espace structurant dans leur socialisation. L'établissement scolaire est alors véritablement l'objet de leur recherche: l'article de Leïla Frouillou analyse ainsi l'organisation fonctionnelle de l'école maternelle et son rôle dans la socialisation des enfants et le texte de Manon Raimbault et de Catherine Lavandier porte sur les représentations des élèves des ambiances sonores de leur collège.

Les auteurs soulignent les avantages à mener une recherche en milieu scolaire et en même temps les différents problèmes méthodologiques et/ou déontologiques que cela peut poser (N. Oppenchaim, A.L. Le Guern et J.F. Thémines, L. Frouillou, E. Schneider). Les chercheurs doivent se faire accepter aussi bien du corps enseignant que des élèves pour qui le statut du chercheur n'est pas toujours clair. En ayant recours à ces institutions, il existe une possible confusion entre la sphère scolaire et celle de la recherche, confusion qui peut être plus problématique quand il s'agit d'un travail auprès d'adolescents qui entretiennent un rapport conflictuel avec l'institution ou bien quand les élèves veulent "bien faire " et agissent comme si le travail était évalué et noté. Le chercheur doit faire accepter sa présence et en même temps il est impératif que les élèves comprennent son statut et ses objectifs. La place du chercheur auprès des élèves est différente selon qu'il apparaît comme un intervenant extérieur, en réalisant des ateliers avec les enfants (Anna Dewaele, Catherine Lavandier et Manon Raimbault) ou bien se place en observateur dans la salle de classe mais n'intervient pas directement (Elisabeth Schneider) ou encore quand l'activité est réalisée conjointement avec les enseignants lors de sorties scolaires comme c'est le cas pour les "parcours 
iconographiques " (Anne-Laure Le Guern et Jean-François Thémines). À propos de la réalisation de parcours iconographiques, ces auteurs soulignent la difficulté, voire l'impossibilité à éviter les effets d'imposition liés à la présence d'adultes, alors même que les enfants sont libres de photographier ce qu'ils souhaitent et que les adultes n'interviennent pas dans leur choix. II existe donc une série de difficultés propres au travail de recherche au sein de l'établissement scolaire auxquelles le chercheur doit être attentif et qu'il doit analyser dans l'interprétation de ces résultats.

Ces contributions, dont on a montré un certain nombre de points communs et la façon dont ils font écho aux recherches sur les rapports des enfants et des jeunes au territoire, se répartissent dans plusieurs rubriques. Les Carnets de recherches présentent les résultats d'études interrogeant différentes facettes de la question des territoires des enfants et des jeunes. Dans les Carnets de terrain, les auteurs font état des réflexions éthiques et méthodologiques que leur ont inspirées leurs pratiques de recherche auprès de ce groupe d'enquêtés particulier. On trouvera enfin dans les Carnets de lectures plusieurs comptesrendus d'ouvrages dont certains sont considérés comme des références incontournables des Children's Geographies.

\section{Les Carnets de recherches : différents aspects du rapport des enfants et des jeunes à l'espace}

Cinq textes, rassemblés dans la rubrique Carnets de recherche, présentent des résultats de recherches portant sur différents aspects du rapport des enfants et des adolescents à l'espace. Les trois premiers portent sur des espaces du quotidien de l'enfance et de l'adolescence au-delà du domicile, trois espaces institutionnels : le premier article examine l'école maternelle comme espace de socialisation des enfants (Leïla Frouillou); le deuxième envisage la géographie sonore d'un collège, telle qu'elle est perçue par ses élèves (Catherine Lavandier et Manon Raimbault), tandis que le troisième se penche sur des lieux moins ordinaires et très peu étudiés : les établissements de placement des mineurs (Fleur Guy). Les deux articles suivants interrogent les mobilités des enfants et des jeunes, le premier à travers l'analyse des lieux des pratiques de loisirs des enfants et des jeunes (Olivier David), et le second à travers un examen de l'impact de l'utilisation du téléphone portable et de I'Internet sur les mobilités et sociabilités adolescentes à Rio et en banlieue parisienne (Hélène Pétry).

Cette rubrique s'ouvre sur un article consacré à l'école maternelle, envisagée comme un espace fonctionnel et transitionnel entre deux formes d'organisation normées, la famille et l'école. Leïla Frouillou, à partir d'un travail de terrain dans deux écoles de banlieue parisienne, montre comment l'organisation spatiale fonctionnelle de l'école maternelle est révélatrice du rôle social que lui attribue l'institution, à savoir une socialisation des enfants. Elle utilise une approche foucaldienne en montrant comment l'impératif d'ordre et de placement, sous-tendu par une logique fonctionnelle, permet de réguler le comportement spatial et social des enfants. Une véritable socialisation s'opère par l'espace, des normes socio-spatiales sont instituées comme les rapports ordre/désordre, accès sous conditions, seuils, respect de la fonctionnalité des espaces, etc. L'auteur complète son analyse des normes socio-spatiales de l'école par une étude des pratiques enfantines et montre comment leurs transgressions et les interstices de liberté dont les enfants disposent leur permettent de s'approprier l'espace scolaire et de construire leur autonomie sociale. 
Nous restons dans les établissements scolaires de banlieue parisienne mais en abordant une dimension très différente, celle de l'espace sonore, avec l'article de Catherine Lavandier et Manon Raimbault portant sur trois collèges, qui constituent tout à la fois les cadres et les objets de la recherche. Cet article est le fruit d'une recherche opérationnelle visant à améliorer la qualité des ambiances sonores dans les collèges. Les auteures analysent la façon dont les collégiens appréhendent la dimension sonore dans leur rapport à l'espace et leurs perceptions des ambiances sonores du collège. Quelles significations donnent-ils aux bruits qui les entourent? Dans la lignée de travaux de géographie réalisés pour analyser les paysages sonores (les travaux du CRESSON en particulier), les enfants ont réalisé des cartes mentales sonores et la richesse iconographique des dessins des collégiens révèle ainsi une géographie des enfants inhabituelle, celle des sons et des bruits du collège.

Après l'étude attentive d'espaces dédiés aux enfants et leurs perceptions de ces espaces, l'article de Fleur Guy se situe à une autre échelle spatiale, celle du département (le Rhône) et des principes de localisation des établissements de placement des mineurs. A travers cet exemple, l'auteure propose une réflexion sur les notions de place et de placement des mineurs au sein de la société française. Elle montre comment la mesure de placement touche deux catégories d'individus que les institutions tendent à séparer: d'un côté une enfance identifiée en danger dans son milieu familial, de l'autre une enfance perçue comme menaçante pour la société. Fleur Guy souligne les ambiguïtés liées au statut du mineur placé, victime et/ou coupable pour lequel l'éloignement constitue un moyen de protection et/ou punition. À travers une analyse historique des mesures de placement, elle montre que la rupture que constitue l'éloignement du jeune avec son milieu fut sans cesse présentée comme nécessaire dans la mesure où l'environnement du jeune est en partie considéré comme responsable de ses problèmes. Le rapport à l'espace est ici central dans ce qui apparaît comme une véritable transformation du jeune opérée par un changement de milieu. L'auteure analyse la localisation des établissements qui doivent répondre aujourd'hui à un double objectif de mise à distance d'un milieu (familial et/ou social) et de proximité, car cette prise en charge doit aussi garantir une proximité entre le jeune et sa famille et l'accessibilité des structures nécessaires à son insertion.

Au delà de l'analyse d'un lieu en particulier, Olivier David s'attache quant à lui à étudier l'articulation des lieux majeurs qui fondent la territorialité quotidienne des enfants et des jeunes (parmi lesquelles on retrouve le domicile et l'établissement scolaire, mais aussi les espaces de loisirs, les lieux de rencontres avec les pairs). À partir d'une vaste enquête menée en Ille-et-Vilaine, il s'intéresse à l'inscription spatiale des pratiques d'activités socioéducatives des enfants et des jeunes durant leur temps libre et aux mobilités qu'elles impliquent, pour les enfants ainsi que pour leurs parents, souvent contraints de les accompagner. Ainsi les différents espaces pratiqués et leur articulation constituent les territoires du quotidien des enfants et des jeunes, mais plus largement aussi les territoires du quotidien familial. La localisation et les mobilités engendrées par la pratique d'activités sont centrales dans l'organisation de la vie quotidienne des familles. Les résultats de l'enquête menée montrent que les pratiques de loisirs des enfants et des jeunes diffèrent selon leur lieu de résidence et leur environnement social et culturel, et qu'avec l'âge des enfants et la spécialisation des activités, l'espace des pratiques de loisirs s'élargit sensiblement et participe à l'autonomie progressive des enfants et des jeunes en matière de mobilité. 
Hélène Pétry traite également des questions d'autonomie spatiale et de mobilités des jeunes, dans son article comparatif sur les mobilités de lycéens de Rio de Janeiro et de la région parisienne. En effet, dans sa contribution, elle analyse l'imbrication de l'espace numérique et de l'espace physique de sociabilité. À l'instar d'autres études sociologiques françaises sur le rôle des sociabilités numériques dans le renforcement et l'autonomisation du groupe de pairs (Delaunay-Téterel, 2010 ; Jarrigeon et Menrath, 2010 ; Metton, 2010 ; Pasquier, 2005), Hélène Pétry démontre que la forte connectivité numérique des adolescents (téléphone portable et Internet) favorise leur mobilité spatiale et leurs sociabilités. Elle analyse le lien entre « joignabilité » et autonomie spatiale et montre que les adolescents franciliens gagnent en mobilité spatiale du fait qu'ils sont toujours joignables par leur famille. Leurs liens internes et leur autonomie par rapport aux adultes sont renforcés par les communications numériques. Le téléphone portable joue une autre fonction pour les jeunes cariocas pour qui il permet de concilier solidarité familiale et mobilité spatiale. L'auteure montre dans cet article comment la joignabilité dans l'espace numérique et les communications, loin de se substituer aux rencontres en présence, les favorisent en multipliant les contacts et en facilitant l'organisation des déplacements. Son texte rejoint par certains aspects, les réflexions menées par Elisabeth Schneider dans ce numéro sur les digital natives et les pratiques d'écriture des adolescents, notamment à travers les réseaux sociaux et les SMS, et l'article de Nicolas Oppenchaim qui aborde également le thème des mobilités d'adolescents de banlieue parisienne mais en s'interrogeant, lui, sur les méthodes et les enjeux d'une recherche menée auprès de jeunes.

\section{Les Carnets de terrain : quelles méthodes et quels enjeux propres à l'élaboration d'une géographie des enfants et des jeunes?}

Un second ensemble de quatre textes, regroupés dans la rubrique Carnets de terrain, porte sur la question des méthodes déployées pour réaliser des enquêtes auprès d'enfants et d'adolescents, sur l'interaction enquêteur/enquêté et les conséquences de la distance générationnelle, sur la question du " consentement éclairé » des enquêtés et sur les enjeux éthiques liés à une recherche auprès d'enfants ou d'adolescents, qui forment un groupe social plus vulnérable que les adultes. À travers ces Carnets de terrain, des exemples concrets de dispositifs méthodologiques mis en œuvre auprès d'enfants sont présentés et analysés dans une perspective réflexive. Les articles offrent ainsi des pistes pour répondre à deux interrogations :

- Est-ce que prendre les enfants ou les adolescents comme objet d'étude suppose I'utilisation de méthodes de recherche différentes de celles utilisées pour les adultes?

- Quelles sont les méthodes permettant de concilier rigueur scientifique et préoccupations déontologiques?

Les contributions font état de diverses méthodes d'enquêtes et focales d'analyse qui permettent de saisir les enjeux spécifiques d'une recherche menée auprès d'enfants. Cette rubrique s'ouvre sur un article consacré aux interrogations méthodologiques et éthiques suscitées par une recherche participative menée sur les pratiques de mobilité d'adolescents habitant des quartiers défavorisés (Nicolas Oppenchaim). Plusieurs articles s'intéressent ensuite à différents outils de recherche permettant de saisir la perspective des enfants euxmêmes, certains relativement classiques, et d'autres moins usuels : le dessin et les cartes 
mentales (Anna Dewaele), mais aussi l'écriture (Elisabeth Schneider), et le "parcours iconographique" (Anne Le Guern et Jean-François Thémines).

La rubrique s'ouvre sur un article de Nicolas Oppenchaim présentant un exemple de participation active d'adolescents de ZUS de banlieue parisienne à une recherche sur leurs mobilités urbaines, recherche à laquelle les jeunes ont véritablement été associés puisqu'ils ont eux-mêmes réalisé des questionnaires qu'ils ont distribués à d'autres jeunes, pris des photographies et écrit des textes sur leurs mobilités, avant d'être interrogés individuellement sur ce thème par le chercheur. Cette méthode participative, innovante mais relativement répandue dans les recherches anglo-saxonnes, comporte de nombreux avantages éthiques et scientifiques : elle favorise le consentement éclairé des adolescents en leur faisant comprendre les implications de leur participation. Elle permet également de créer une relation de confiance avec les adolescents et de stimuler leur réflexivité afin de les associer à l'élaboration de la recherche. Mais elle pose aussi certains problèmes pour le chercheur qui expose dans cet article les difficultés qu'il a rencontrées pour mettre en œuvre cette méthode et la façon dont il a dû reformuler sa méthode pour gérer les imprévus.

Anna Dewaele présente elle aussi son expérience de recherche, auprès d'écoliers à Chandigarh en Inde. Elle a réalisé un atelier dans une école primaire auprès de quelques enfants afin de questionner leurs représentations de cette ville nouvelle: ils devaient dessiner leur ville telle qu'elle est aujourd'hui et telle qu'ils l'imaginent demain. Elle explique comment ce travail avec les enfants, qui avait d'abord une valeur comparative avec les représentations de citadins adultes, a considérablement nourri sa réflexion sur les représentations urbaines de Chandigarh. Dans ce texte, elle expose les modalités de cet atelier, réalisé à titre expérimental, et ce qu'elle en a tiré pour de futures recherches. Elle donne ainsi des éléments pratiques et concrets sur le déroulement de l'atelier, l'attitude des enfants et leurs réactions vis-à-vis de l'exercice. Plus que le dessin en lui-même, le moment de la réalisation est un moment privilégié de dialogue entre le chercheur et les enfants sur leur ville. Le dessin devient autant un produit à analyser que le prétexte ou le support à la discussion. Il permet d'engager, à partir de quelque chose de concret et tangible, un dialogue sur les représentations urbaines enfantines, difficilement saisissables par le biais d'entretiens.

L'article d'Elisabeth Schneider porte sur d'autres outils méthodologiques; elle nous livre, dans une perspective réflexive, ses interrogations sur la manière dont il est possible d'accéder aux pratiques d'écriture adolescentes : à travers les écrits entre pairs, hors et dans la classe, sur les réseaux sociaux numériques. Elle évoque la nécessité d'instaurer une relation de confiance avec les adolescents afin qu'ils montrent des écrits qu'ils cachent parfois aux adultes. Son objectif est de suivre des adolescents dans leurs déplacements et leurs activités, dans la sphère scolaire et privée, mais aussi de collecter tous les écrits produits par les adolescents, quels qu'ils soient. Elle souhaite ainsi les inscrire dans un contexte de production et entendre ce que ces adolescents ont à en dire pour percevoir la complexité et le rôle de ces écrits dans la production des espaces. Elle a choisi certaines situations fréquemment vécues par les adolescents qui condensaient leurs relations à l'espace par et avec l'utilisation de l'écriture. Dans une démarche ethnographique, elle combine observations en classe, entretiens et analyses des écrits adolescents à partir des 
" mots » qui circulent dans la salle de cours, des SMS ou des forums sociaux comme Facebook.

Enfin, l'article d'Anne-Laure Le Guern et Jean-François Thémines présente la méthodologie du " parcours iconographique ", une méthode de recherche innovante qui permet de rendre compte d'une expérience spatiale enfantine de la ville. Cette méthode est illustrée par l'exemple concret d'une sortie de classe d'enfants de primaire scolarisés à Lisieux. Les auteurs expliquent en quoi cette méthode se rapproche et se distingue de celle du " parcours commenté " (Thibaud, 2001), qui inclut elle aussi la pratique de parcours ou la production et/ou le commentaire de photographies. L'objectif des auteurs est de mieux connaître les ressorts de l'expérience urbaine des enfants et de cerner leur capacité à produire un discours sur cet espace public proche. À travers leurs photographies et leurs textes dont les auteurs présentent des exemples, les enfants expriment leur représentation d'un espace urbain qu'ils connaissent partiellement par leurs pratiques quotidiennes. Les auteurs expliquent comment cette méthode permet de construire une géographie des enfants faite par eux, tout en n'étant pas spontanée, car elle est expérimentée en articulation avec le milieu scolaire. Les enfants réalisent librement des photographies : les adultes encadrant le groupe d'enfants n'exercent pas de contrainte sur le rythme, l'angle de vue, le cadrage des photographies ainsi que les paysages, scènes ou objets photographiés. Le parcours iconographique place les enfants en position de production d'une iconographie sur la ville : ils sont ainsi considérés comme des producteurs de savoir originaux et légitimes.

\section{Les Carnets de lecture : quelques références pour les géographies des enfants et des jeunes}

La rubrique Carnets de lecture complète les contributions de ce numéro spécial sur les géographies des enfants et des jeunes. A côté de la recension d'un ouvrage français, cinq comptes-rendus portent sur des livres anglophones, dont certains sont déjà un peu anciens (début des années 2000) mais constituent des ouvrages de référence des Children's Studies anglophones alors qu'ils sont encore peu connus dans le milieu scientifique francophone.

Enfin, on pourra lire le Carnet de lecture proposé par Leïla Frouillou, qui s'éloigne du modèle canonique du compte-rendu (comme le promeut la ligne éditoriale de la revue) puisqu'il s'agit d'une réflexion sur l'usage des albums jeunesse pour une géographie des enfants: l'auteure y interroge en particulier les différents types de représentations de la maison dans la littérature enfantine.

\section{Bibliographie}

AITKEN S. C. (1994), Putting Children in their Place, Washington, Association of American Geographers.

AITKEN S. C. (2001), Geographies of Young People: The Morally Contested Spaces of Identity, Londres, Routledge.

ALDERSON P. et MORROWS P. (2004), Ethics, Social Research and Consulting with Children and Young People, Londres, Barnardos.

ANDRE Y., BAILLY A., FERRAS R., GUERIN J.-P. et GUMUCHIAN H. (dir.) (1989), Représenter l'espace : L'imaginaire spatial à l'école, Paris, Anthropos. 
ARIES P. (1962), L'enfant et la vie familiale sous l'Ancien Régime, Paris, Plon.

AUTHIER J.-Y. et LEHMAN-FRISCH S. (2012), " II était une fois... des enfants dans des quartiers gentrifiés à Paris et à San Francisco ", Actes de la Recherche en Sciences Sociales (à paraître).

BEAUD S. (2002), 80\% au bac... et après? Les enfants de la démocratisation scolaire, La Découverte, Paris.

BERTRAND M.J. et METTON A. (1972), «La perception de l'espace urbain: de l'enfant à I'homme ", L'espace géographique, 1-4, pp. 283-285.

BLAUT J. M. et STEA D. (1971), "Studies of Geographic Learning ", Annals of the Association of American Geographers, 61, pp. 387-393.

BLAUT J. M., STEA D., SPENCER C. et BLADES M. (2003), "Mapping as a Cultural and Cognitive Universal », Annals of the Association of American Geographers, 93-3, pp. 165-185.

BONIN S. (2004), « Au-delà de la représentation, le paysage », Strates, 11.

BUNGE W. (1973), "The Geography », The Professional Geographer, 25-4, pp. 331-337.

CALLENGE C., LUSSAULT M., PAGAND B. (dir.) (1997), Figures de l'urbain: Des villes, des banlieues et de leurs représentations, Tours, Maison des Sciences de la Ville.

CASABIANCA R. M. de (1959), Enfants sans air : Etude sociologique des enfants d'un quartier urbain, Paris, Fleurus.

CELE S. (2006), Communicating Place: Methods for Understanding Children's Experience of Place, Acta Universitiatis Stockholmiensis, Stockholm Studies in Human Geography, Stockholm University.

CHAWLA L. (ed.) (2002), Growing Up in an Urbanizing World, Londres, UNESCO, Earthscan Publications.

CHOMBART DE LAUWE M.-J. (1977), Dans la ville, des enfants, Paris, Autrement, 10.

CHOMBART DE LAUWE M.-J. (1987), Espaces d'enfants, la relation enfant-environnement, ses conflits, Delval.

CHRISTENSEN P., JAMES A. et JENKS C. (2000), "Home and Movement: Children Constructiong 'Family Time' ", in HOLLOWAY S., VALENTINE G., Children's Geographies: Playing, Living, Learning, London, Routledge, pp. 139-155.

CHRISTENSEN P. et O'BRIEN M. (eds) (2003), Children in the City: Home, Neighbourhood and Community, Londres, Routledge.

CLOUTIER M.-S. et TORRES J. (dir.) (2010), "L'enfant et la ville : Notes introductoires", Enfances Familles, Générations, 12, pp. i-xv.

COLLS R. et HORSCHELMANN K. (2009), "The Geographies of Children's and Young People's Bodies ", Children's Geographies, 7-1, pp. 1-6.

DANIC I., DAVID O. et DEPEAU S. (dir.) (2010), Enfants et jeunes dans les espaces du quotidien, Rennes: Presses Universitaires de Rennes. 
DANIC I., DELALANDE J. et RAYOU P. (2006), Enquêtes auprès d'enfants et de jeunes : Objets, méthodes et terrains de recherche en sciences sociales, Presses Universitaires de Rennes.

DAVID O. (2010), "Introduction générale », in DANIC I., DAVID O. et DEPEAU S. (dir.), Enfants et jeunes dans les espaces du quotidien, Rennes, Presses Universitaires de Rennes, pp. 7-13.

DELALANDE J. (2001), La cour de récréation: Pour une anthropologie de l'enfance, Rennes, PUR.

DELALANDE J. (2003), La récré expliquée aux parents, Paris, Audibert.

DELALANDE J. (2010), "La socialisation des enfants dans la cour d'école: une conquête consentie ? ", in DANIC I., DAVID O. et DEPEAU S. (dir.), Enfants et jeunes dans les espaces du quotidien, Rennes, Presses Universitaires de Rennes, pp. 35-48.

DELAUNAY-TETEREL, H. (2010), " L'affichage public des amitiés: Le blog au lycée ", Ethnologie française, $\mathrm{XL} / 1, \mathrm{pp} .115-122$.

DEPEAU S. (2003), L'enfant en ville: Autonomie de déplacement et accessibibilité environnementale, Thèse de doctorat en psychologie, Université R. Descartes-Paris 5.

DEPEAU S. (2008), « Radioscopie des territoires de la mobilité des enfants en milieu urbain : Comparaison entre Paris intra-muros et banlieue parisienne ", Enfances, Familles, générations, $n^{\circ} 8$.

DE SINGLY F. (1998), Habitat et relations familiales, Paris, Plan construction et architecture.

ENAUX C. et LEGENDRE A. (2010), “ Méthode d'identification des lieux investis par des enfants de six à onze ans dans leur espace de vie urbain quotidien ", DANIC I., DAVID O. et DEPEAU S. (dir.), Enfants et jeunes dans les espaces du quotidien, Rennes: Presses Universitaires de Rennes, pp. 89-100.

FERRAS R. (1978), “'Ecusson' et 'polygone': Enfants et retraités dans le centre de Montpellier ", Bulletin de la Société Languedocienne de Géographie, 101-1, Montpellier.

FREMONT A. (1976), La région, espace vécu, Paris, Flammarion, 1999 ( $2^{\text {ème }}$ édition).

GALLAND O. (2006), Sociologie de la jeunesse, Paris, Armand Colin.

GOULD P. et WHITE R. (1974), Mental Maps, Harmondsworth, Penguin Books.

GUREVITZ R. et SANGUIN A.-L., "Les géographies des enfants: un survol de la recherche britannique, 1970-2000 ", Géographie et Cultures, n³9, 2001, pp. 116-123.

HALLDEN G. (2003), "Children's Views of Family, Home and House ", in CHRISTENSEN P., O'BRIEN M. (eds), Children in the City: Home, Neighbourhood and Community, London, Routledge, pp. 29-45.

HART R. (1979), Children's Experience of Place : A Developmental Study, New York, Irvington Publishers.

HATFIELD M. E., (2010) « Children moving 'home'? Everyday Experiences of Return Migration in Highly Skilled Households », Childhood 17- 2, pp. $243-257$.

HOLLINGWORTH S., WILLIAM S., JAMIESON F. et BEEDELL P. (2011), « Social and Educational Inequalities in English State Schools : Exploring the Understandings of Urban White Middle- 
Class Children ", in HOLT L. (ed.), Geographies of Children, Youth \& Families, Londres et New York, Routledge, pp. 250-264

HOLLOWAY S. et VALENTINE G. (2000), Children's Geographies: Playing, Living, Learning, Londres, Routledge.

HOLLOWAY S. et PIMLOTT-WILSON H. (2010), "Geographies of Children, Youth and Families : Defining Achievements, Debating the Agenda ", in HOLT L. (ed.), Geographies of Children, Youth \& Families, Londres et New York, Routledge, pp. 9-24.

HOLT L. (ed.) (2011), Geographies of Children, Youth \& Families, Londres et New York, Routledge.

HOLT L. et COSTELLO L. (2011), "Beyond Otherness: Exploring Diverse Spatialities and Mobilities of Childhood and Youth Populations ", Population, Space and Place, 17-4, pp. 299303.

HOPKINS P. E. (2010), Young People, Place and Identity, Londres et new York, Routledge.

HOPKINS P. E. et HILL M. (2008), «Pre-flight Experiences and Migration Stories: The Accounts of Unaccompanied Asylum-seeking Children ", Children's Geographies, 6-3, pp. 257-268.

HOPKINS P. E. et PAIN R. (2007), « Geographies of Age : Thinking Relationally », Area, 39, pp. 287-294.

HORTON J., KRAFTL P. et TUCKER F. (2008), " The Challenges of 'Children's Geographies' : An Affirmation ", Children's Geographies, 4-1, pp. 69-98.

JARRIGEON A. et MENRATH J. (2010), « De la créativité partagée au chahut contemporain. Le téléphone mobile au lycée », Ethnologie française, XL/1, pp. 109-114.

JENKS C. (1996), Childhood, Londres, Routledge.

KARSTEN L. ET VAN VILET W. (2006), «Increasing Children's Freedom of Movement: Introduction ", Children, Youth and Environment, 16-1, pp. 69-73.

KATZ C. (2004), Growing Up Global : Economic Restructuring and Children's Everyday Lives, Minneapolis, University of Minnesota Press.

KOKOREFF M. (2003), La Force des quartiers. De la délinquance à l'engagement politique, Paris, Payot.

KRAFTL P. (2006), "Building an Idea: The Material Construction of an Ideal Childhood", Transactions of the Institute of British Geographers, $n^{\circ} 31$, pp. 488-504.

LAREAU A. (2003), Unequal Childhoods: Class, Race and Family Life, Berkeley, Los Angeles, Londres, University of California Press.

LEGENDRE A. (2010), "Evolution de la connaissance et de l'utilisation des espaces publics extérieurs entre 6 et 11 ans : le cas d'Arpajon, une petite ville de la banlieue parisienne ", in DANIC I., DAVID O. et DEPEAU S. (dir.), Enfants et jeunes dans les espaces du quotidien, Rennes, Presses Universitaires de Rennes, pp. 75-88.

LEPOUTRE D. (2001), Coeur de banlieue, Paris, O. Jacob. 
LIGNIER W. (2010), "Des chambres intelligentes? Un regard sociologique sur l'espace personnel des enfants dits "intellectuellement précoces" ", in DANIC I., DAVID O. et DEPEAU S. (dir.), Enfants et jeunes dans les espaces du quotidien, Rennes, Presses Universitaires de Rennes, pp. 119-128.

LUGADET-AGRAZ J.-B. (1989), "Représentations enfantines des l'espace urbain : Exemples clermontois ", in ANDRE Y., BAILLY A., FERRAS R., GUERIN J.-P. et GUMUCHIAN H. (dir.), Représenter l'espace: L'imaginaire spatial à l'école, Paris, Anthropos, pp. 179-190.

LYNCH K. (1960), The Image of the City, Cambridge, Massachussetts, MIT Press.

LYNCH K. (dir.) (1977), Growing Up in Cities, Cambridge, Massachussetts, MIT Press.

MASSON-VINCENT M. (1995), L'enfant et la montagne: Savoirs géographiques et représentations spatiales sur la montagne, Paris, Anthropos.

MATTHEWS H. (1984), " Cognitive Maps of Young Children : A Comparison of Graphic and Iconic Techniques ", Area, 16, pp. 31-41.

MATTHEWS H. (1985), "Young Children's Representation of the Environment: A Comparison of Techniques ", Journal of Environmental Psychology, 5, pp. 262-278.

MATTHEWS H. (1992), Making Sense of Place: Children's Understandings of Large-Scale Environment, Hemel Hempstead, Harvester Wheatsheaf.

MATTHEWS H. et LIMB M. (1999), "Defining and Agenda for the Geography of Children: Review and Propspect ", Progress in Human Geography, 23-1, pp. 61-90.

MCKENDRICK J. H. (2000), "The Geography of Children: An Annotated Bibliography ", Childhood, 7-3, pp. 359-387.

METTON A. (1976), L'Espace vécu par les enfants de Courbevoie, Paris, CNAC GeorgesPompidou, $\mathrm{CCl}$.

METTON C. (2010), " L'autonomie relationnelle. SMS, "chat" et messagerie instantanée ", Ethnologie française, XL/1, pp. 101-107.

MORELLE M. (2007), La rue des enfants, les enfants des rues, Paris, CNRS éditions.

NEITZERT (1990), La chambre d'enfant : représentations et pratiques qui en affectent l'usage dans une catégorie sociale en évolution, les professions intermédiaires, Recherche à la demande du Plan et Construction, Ministère de l'Equipement, du Logement de l'Aménagement du Territoire et des Transports, Fédération nationale des écoles des parents et des éducateurs, Paris.

O'BRIEN M. (2003), "Regenerating Children's Neighbourhoods : What Do Children Want ? ", in CHRISTENSEN P. et O'BRIEN M. (eds), Children in the City: Home, Neighbourhood and Community, Londres, Routledge, pp. 142-161.

PAIN R. (2004), "Social Geography: Participatory Research», Progress in Human Geography, 30, pp. 652-663.

PASQUIER, D. (2005), Cultures lycéennes, la tyrannie de la majorité, Paris, Autrement, 180p.

PIAGET J. et INHELDER B. (1982), La représentation de l'espace chez l'enfant, Paris: Presses Universitaires de France, 4ème édition. 
PROUT A. et JAMES A. (1990), "A New Paradigm for the Sociology of Childhood? Provenance, Promise and Problems ", in JAMES A. et PROUT A. (eds), Constructing and Reconstructing Childhood, Londres, Falmer Press.

RAMADIER T. et DEPEAU S. (2010), " Approche méthodologique (JRS) et développementale de la représentation de l'espace quotidien de l'enfant», in Danic I., David O. et Depeau S. (dir.), Enfants et jeunes dans les espaces du quotidien, Rennes, Presses Universitaires de Rennes, 2010, pp.61-74.

RAYOU P. (2006), "Ni tout à fait les mêmes, ni tout à fait autres. Les territoires et calendriers scolaires des enfants et des jeunes ", in SIROTA R. (dir.), Eléments pour une sociologie de l'enfance, Presses universitaires de Rennes, pp. 217-224.

RENAHY N. (2005), Les gars du coin : Enquête sur une jeunesse rurale, Paris, La Découverte.

SGARD A. et HOYAUX A.-F. (2006), "L'élève et son lycée: De l'espace scolaire aux constructions des territoires lycéens ", L'Information géographique, vol. 70, pp. 87-106.

SIBLEY D. (1995), "Families and Domestic Routines: Constructing the Boundaries of Childhood ", in PILE S. et THRIFT N., Mapping the Subject: Geographies of Cultural Transformation, London, Routledge, pp. 123-139.

SIROTA R. (1998), "Les copains d'abord: Les anniversaires de l'enfance, donner et recevoir ", Ethnologie française, 28-4, pp. 457-471.

SIROTA R. (dir.) (2006), Eléments pour une sociologie de l'enfance, Rennes, Presses Universitaires de Rennes.

SMITH F. et BARKER J (2000), "'Out of school', in School: A Social Geography of Out of School Children ", in HOLLOWAY S. et VALENTINE G. (dir.), Children's Geographies: Playing, Living, Learning, Londres, Routledge, pp. 245-257.

TESSIER S. (dir.) (2005), L'enfant des rues, Contribution à une socio-anthropologie de l'enfant en grande difficulté dans l'espace urbain, Paris, L'Harmattan.

THIBAUD J.-P. (2001), "La méthode des parcours commentés ", in GROSJEAN M., THIBAUD J.-P. (dir.), L'espace urbain en méthodes. Marseille, Editions Parenthèses, pp.79-100.

TSOUKALA T. (2007), les territoires urbains de l'enfant, Paris, L'Harmattan.

VALENTINE G. (2004), Public Space and the Culture of Childhood, London, Ashgate.

VAN BLERK L. et ANSELL N. (2006), « Imagining Migration: The Impact of Place on Children's Understanding of 'Moving House' in Southern Africa », Geoforum 37-2, pp. 256-272.

WARD C. (1978), The Child in the City, Londres, Architectural Press, 1978.

WHITE A., BUSHIN N., CARPENA-MENDEZ F. et NI LAOIRE C. (2010), « Using Visual Methodologies to Explore Contemporary Irish Childhoods ", Qualitative Research 10-2, pp. $143-158$.

WOODYER T. (2008), "The Body as Research Tool: Embodied Practice and Children's Geographies ", Children's Geographies, 6-2, pp. 349-362. 